PROCEEDINGS OF THE

AMERICAN MATHEMATICAL SOCIETY

Volume 132, Number 5 , Pages 1367-1375

S 0002-9939(03)07064-3

Article electronically published on December 22, 2003

\title{
A CONTINUED FRACTION ANALYSIS OF PERIODIC WAVELET COEFFICIENTS
}

\author{
JOEL GLENN
}

(Communicated by David R. Larson)

\begin{abstract}
We define and prove the existence of crossings of wavelet coefficients translated by integer multiples of the numerator of a continued fraction convergent of the ratio of the sampling interval to the period of the wavelet coefficients. Crossings are found to be translation invariant \pm 1 . Intervals between crossings are analyzed for wavelets with $n$ vanishing moments. These wavelets act as multiscale differential operators. These crossings reveal different locations in the period where there is equality in the $n$th derivative of an averaging of the signal. These results will be employed in the estimation of frequency components in future publications.
\end{abstract}

The following constitutes part of the author's dissertation under the direction of Arlan Ramsay at the University of Colorado at Boulder. Wavelet coefficients of periodic signals have been the subject of previous study [1]. This paper is a further analysis of the patterns of wavelet coefficients of periodic signals. A possible application of this is the efficient isolation and analysis of periodic components of a signal. Now, if the ratio between the period and the sampling interval is rational, the wavelet coefficients will simply repeat after a whole number of periods have been processed. However, if the ratio is irrational such repetition will not occur. Instead, the coefficients will exhibit patterns associated with the continued fraction convergents of this ratio. For the purposes of this paper data is assumed to be discrete from a continuous signal $f$ with period $P$. The wavelets are assumed to decay rapidly so the wavelet coefficients exist. Let $I$ be the interval between samples. Assume $P$ is an irrational multiple of $I$. Wavelet coefficients will be computed for a fixed level of resolution $2^{j}, j \in \mathbb{Z}$. Let $M=\frac{2^{j} P}{I}$. Let $\left\{\frac{p_{k}}{q_{k}}\right\}_{k \in \mathbb{N}}$ be the set of continued fraction convergents for $M$. The proposition below constructs a sequence of wavelet coefficients which converges to an initial, untranslated coefficient.

Proposition 1. Let $\psi$ be a wavelet with rapid decay. Let $P, M, I, p_{k}, q_{k}$, and $f(x)$ be as above. Then

$$
\lim _{k \rightarrow \infty} \int_{-\infty}^{\infty} \psi\left(2^{j} x-l-p_{k} I\right) f(x) d x=\int_{-\infty}^{\infty} \psi\left(2^{j} x-l\right) f(x) d x
$$

for all $l \in \mathbb{Z}$.

Proof. In the first integral, change the variable as follows:

$$
u=2^{j} x-l-p_{k} I \text {. }
$$

Received by the editors February 19, 2002 and, in revised form, September 26, 2002. 2000 Mathematics Subject Classification. Primary 42C40, 65T60; Secondary 11A55, 40A15. 
We obtain

$$
\int_{-\infty}^{\infty} \psi(u) f\left(2^{-j}\left(u+l+p_{k} I\right)\right) \frac{d u}{2^{j}} .
$$

Now,

$$
\begin{aligned}
2^{-j} & \left|\int_{-\infty}^{\infty} \psi(u)\left[f\left(2^{-j}\left(u+l+p_{k} I\right)\right)-f\left(2^{-j}(u+l)\right)\right] d u\right| \\
& =2^{-j}\left|\int_{-\infty}^{\infty} \psi(u)\left[f\left(2^{-j}\left(u+l+p_{k} I\right)\right)-f\left(2^{-j}\left(u+l+q_{k} 2^{j} P\right)\right)\right]\right| .
\end{aligned}
$$

This follows from the $P$ periodicity of $f$. Observe that

$$
\begin{aligned}
2^{-j}\left|u+l+p_{k} I-u-l-q_{k} 2^{j} P\right| & =2^{-j}\left|p_{k} I-q_{k} 2^{j} P\right| \\
& =2^{-j}\left|q_{k}\right|\left|\frac{p_{k}}{q_{k}} I-2^{j} P\right| \\
& <2^{-j} \frac{q_{k}|I|}{q_{k}^{2}}=\frac{|I|}{2^{j} q_{k}} .
\end{aligned}
$$

The last inequality follows from a well-known theorem in the theory of continued fractions. See [2]. Since $f$ is continuous, we can choose $k$ so large that the integral is less than

$$
2^{-j}\left|\int_{-\infty}^{\infty} \psi(u) \epsilon d u\right| \leq 2^{-j} \epsilon \int_{-\infty}^{\infty}|\psi(u)| d u,
$$

for any $\epsilon>0$.

We now define the main concept of this paper.

Let $\psi$ be a wavelet with rapid decay. Let

$$
W_{d} f(j, x)=2^{\frac{j}{2}} \int_{-\infty}^{\infty} \psi\left(2^{j} t-x\right) f(t) d t .
$$

Definition 2. Let $P, M, I, p_{k}, q_{k}$, and $f$ be as above and $j \in \mathbb{Z}$ remain fixed. For $r, s \in \mathbb{N}$ let

$$
g(x)=W_{d} f(j, r I+x)-W_{d} f(j, s I+x), \text { for } x \in \mathbb{R} .
$$

Let $t \in \mathbb{N}$. We say $r$ and $s$ have a crossing of order $k$ at $t$ if

$$
\operatorname{sign}\left(g\left(t p_{k} I\right)\right)=-\operatorname{sign}\left(g\left((t+1) p_{k} I\right)\right)
$$

or if

$$
g\left(t p_{k} I\right)=0 .
$$

The above definition and the results that follow explain phenomena that can actually be observed when examining the wavelet coefficients of a periodic function where $M$ is irrational (see the image below). This paper will concentrate on wavelets with vanishing moments. It is known that wavelets with $n$ vanishing moments act as an $n t h$ order multiscale differential operator. That is, the signal is convolved with an averaging function and the $n t h$ derivative is taken. These coefficients converge to the $n t h$ derivative of the signal as the level of resolution increases. For a discussion of these facts see [3].

We now give the meaning of the crossings for wavelets with $n$ vanishing moments. The crossing of two strands of coefficients reveals two locations in the period where the $n t h$ derivative of the averaged signal is equal. Two locations are revealed 


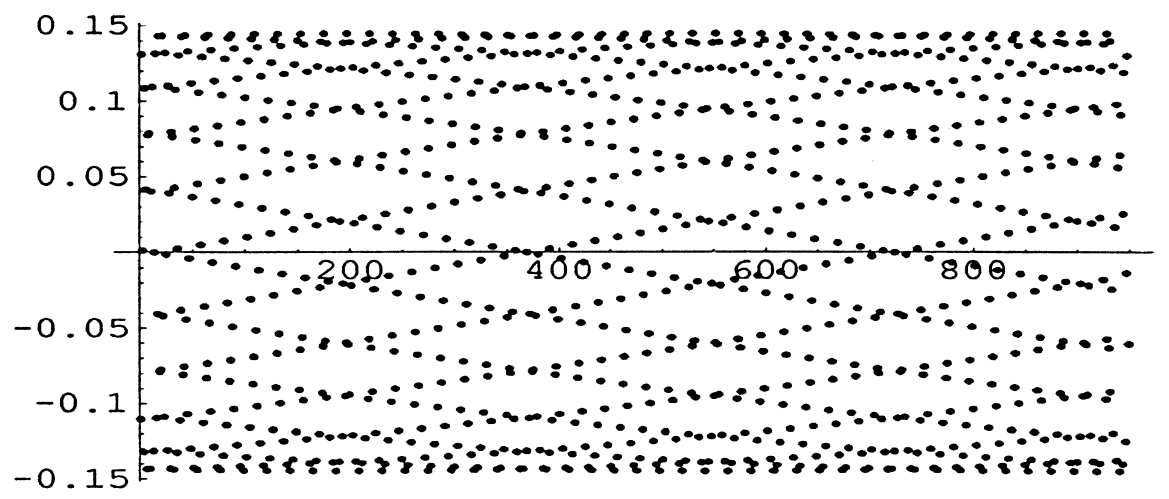

FIGURE 1.

because the strands begin with different translation values, $r I$ and $s I$. Fortunately, the crossings are predictable in that the interval between crossings and the number of crossings in each period are nearly translation invariant. Since the crossings are nearly translation invariant it is possible that by retaining only the coefficients near the crossings and recording the length of the interval between them one may retain much of the structure of the signal. Variations over time in the length between these crossings will reveal variations in the internal derivative structure of the signal. This is a subject for future research. Rationale for studying these phenomena therefore include what crossings reveal about the derivatives of a signal and the possible future development of compression algorithms.

Each strand of coefficients can be identified since adjacent coefficients in a strand will be locally the closest coefficients to one another. This is because each continued fraction coefficient of $M$ is a best approximation of $M$. If the signal is periodic, these patterns of closest approach at order $k$ will correspond to the numerator, $p_{k}$, of a continued fraction convergent of $M$. In the case where $M$ is rational, strands will each become horizontal once the order is sufficiently high and no crossings will be observed. An algorithm for identifying strands from a set of coefficients in the irrational case has been developed and will be the subject of future publications. The image in Figure 1 is a set of wavelet coefficients of $\sin x$ where the analyzing wavelet is the quadratic Battle-Lemarié wavelet. This wavelet has two vanishing moments. Here $I=1, j=-1, P=2 \pi$, and $M=\frac{2^{-1} 2 \pi}{1}=\pi$. The visible strands correspond to $p_{k}=22$ and $q_{k}=7$ so that adjacent coefficients in a strand are 22 coefficients apart. The crossings are of order $k=2$. Other crossings are present but not visually prominent. Figure 1 was generated by Wavelet Explorer by Mathematica.

Now we prove the existence of the crossings in the irrational case. Note that crossings exist for all orders $k$ above a certain value.

Theorem 3. Let $P, M, I, p_{k}, q_{k}$, and $f$ be as above. Assume also that $W_{d} f(j, x)$ has a finite number of zeroes in each interval of length $2^{j} P$. Then there exists a $K \in \mathbb{N}$ such that for all $k \geq K$, and all $r, s \in \mathbb{N}$ such that $r I, s I<p_{k} I$, there exists at $t \in \mathbb{N}$ such that $r$ and $s$ have a crossing of order $k$ at $t$.

Proof. We first establish the following lemma. 
Lemma 3.1. Let $k \in \mathbb{N}$. Then for all $r, t \in \mathbb{Z}$ there is a $\xi_{k}$ such that $\left|\xi_{k}\right| \in$ $\left(\frac{I}{q_{k}+q_{k+1}}, \frac{I}{q_{k+1}}\right)$ such that

$$
W_{d} f\left(j, r I+t p_{k} I\right)=W_{d} f\left(j, r I+t\left|\xi_{k}\right|\right) .
$$

Proof of Lemma 3.1. For odd $k, \frac{p_{k}}{q_{k}} I>2^{j} P$. See [4].

Now,

$$
\frac{1}{q_{k}\left(q_{k}+q_{k+1}\right)}<\frac{p_{k}}{q_{k}}-M<\frac{1}{q_{k}\left(q_{k+1}\right)} .
$$

Hence,

$$
\frac{I}{q_{k}+q_{k+1}}<p_{k} I-q_{k} 2^{j} P<\frac{I}{q_{k+1}} .
$$

Set $\xi_{k}=p_{k} I-q_{k} 2^{j} P>0$. Then for all $t \in \mathbb{Z}$ we have

$$
W_{d} f\left(j, r I+t p_{k} I\right)=W_{d} f\left(j, r I+t \xi_{k}+t q_{k} 2^{j} P\right) .
$$

Since $t q_{k} \in \mathbb{N}$ we have

$$
W_{d} f\left(j, r I+t \xi_{k}+t q_{k} 2^{j} P\right)=W_{d} f\left(j, r I+t \xi_{k}\right) .
$$

This follows from the periodicity of $f(x)$.

If $k$ is even, the results of the lemma hold with $\xi_{k}<0$ and

$$
\frac{-I}{q_{k+1}}<\xi_{k}<\frac{-I}{q_{k}+q_{k+1}} .
$$

The proof of this is similar to the proof for odd $k$. Here $\xi_{k}<0$ because $k$ is even. For this fact see [4].

Proof of Theorem 3. From Lemma 3.1 it follows that

$$
\left|\xi_{k}\right|>\frac{I}{q_{k}+q_{k+1}} .
$$

Let

$$
T=\left\lceil\left(\frac{\left(q_{k}+q_{k+1}\right) 2^{j} P}{I}\right)\right\rceil .
$$

Then

$$
\begin{aligned}
T\left|\xi_{k}\right| & >\left(\frac{q_{k}+q_{k+1}}{I} 2^{j} P\right)\left(\frac{I}{q_{k}+q_{k+1}}\right) \\
& =2^{j} P .
\end{aligned}
$$

Now, for $k \geq 1$ we have $\left|\xi_{k}\right|<\frac{I}{q_{k+1}}$ and $q_{k} \uparrow \infty$ as $k \rightarrow \infty$. Assume without loss of generality that $r<s$.

Let $g(x)$ be defined as in Definition 2. Assume $g(x)$ is not identically zero. Then $g(x)$ is the difference of translates of a periodic function of period $2^{j} P$. From this we conclude that $g$ has period $2^{j} P$ and also has a zero Riemann integral over each interval of length $2^{j} P$. Since $g$ is continuous we also conclude that it changes sign on any such interval. Further, since $W_{d} f(j, x)$ has only a finite number of zeroes on each interval of length $2^{j} P$, we may select $x_{0}$ such that $g\left(x_{0}\right)=0$ and also such that there exists $\delta>0$ such that for $\left(x_{0}-\delta, x_{0}+\delta\right), g(x)$ has the opposite sign on $\left(x_{0}-\delta, x_{0}\right)$ to that on $\left(x_{0}, x_{0}+\delta\right)$. To see this, suppose there is no such $x_{0}$. 
Then every open interval about $x_{0}$ must contain a zero of $W_{d} f(j, x)$ distinct from $x_{0}$. This contradicts the assumption that $W_{d} f(j, x)$ has only a finite number of zeroes on the intervals of length $2^{j} P$. Choose $K$ so large that $\left|\xi_{K}\right|<\delta$. Then there exists a $t \in \mathbb{N}$ such that $t \xi_{K} \in\left(x_{0}-\delta, x_{0}\right]$ and $(t+1) \xi_{K} \in\left[x_{0}, x_{0}+\delta\right)$. Clearly such a $t$ exists for any $k \geq K$ since $\left|\xi_{k}\right|$ monotonically decreases with $k$. Hence for all $k \geq K$ there exists a $t \in \mathbb{N}$ such that $\operatorname{sign}\left(g\left(t \xi_{k}\right)\right)=-\operatorname{sign}\left(g\left((t+1) \xi_{k}\right)\right)$ or $g\left(t \xi_{k}\right)=0$.

The $T$ given in (18) is strictly larger than the value necessary to guarantee a crossing. A lower bound for $T$ may be computed using the upper bound $\frac{I}{q_{k+1}}$ for $\xi_{k}$ given in Lemma 3.1. Observe that

$$
\begin{aligned}
\left(T-1-\frac{2^{j} P q_{k}}{I}\right)\left(\frac{I}{q_{k+1}}\right) & =\left(\frac{I}{q_{k+1}}\right)\left(\left\lfloor\frac{2^{j} P\left(q_{k}+q_{k+1}\right)}{I}\right\rfloor-\frac{2^{j} P q_{k}}{I}\right) \\
& <2^{j} P\left[\frac{q_{k}+q_{k+1}}{q_{k+1}}\right]-\frac{2^{j} P q_{k}}{q_{k+1}} \\
& =2^{j} P\left[\frac{q_{k+1}}{q_{k+1}}\right] \\
& =2^{j} P .
\end{aligned}
$$

Now, $\frac{2^{j} P q_{k}}{I}=M q_{k}$. M is given by the continued fraction. Therefore, for odd $k$, $\frac{p_{k}}{q_{k}}>M$ and $p_{k}>M q_{k}$. From this it follows that $T-p_{k}-1<T-1-\frac{2^{j} P q_{k}}{I}$. So, we know for odd $k$,

$$
T-p_{k}-1
$$

is too small to guarantee a crossing. A similar argument produces the same lower bound for even $k$. Note that since $f(x)$ is periodic, $g(x)$ will have two zeroes before the $t$ value given in Corollary 4 .

If the wavelet has vanishing moments we can construct a bound for the function $g(x)$ given in Definition 2. The bound is given in terms of the $m$ th derivative of the signal and the $m$ th moment of the wavelet. For the following four results we assume $f$ possesses an $m$ th derivative.

Corollary 4. For $t \geq \frac{\left(q_{k}+q_{k+1}\right)}{I} 2^{j} P$ we have $t\left|\xi_{k}\right|>2^{j} P$.

Proof. This follows from the fact that $\left|\xi_{k}\right|>\frac{I}{q_{k}+q_{k+1}}$ which is given in Lemma 3.1 .

Proposition 5. For a wavelet with $m$ vanishing moments and

$$
K=\frac{1}{2^{j(m+1)}} 2\left\|f^{(m)}\right\|_{\infty}
$$

we have

$$
|g(x)| \leq K\left|M_{m}\right|
$$

Proof. Since $f(x)$ is $m$ times differentiable on $\mathbb{R}$ we may expand about the fixed point $x_{0}$ :

$$
f(x)=\sum_{i=0}^{m-1} f^{i}\left(x_{0}\right) \frac{\left(x-x_{0}\right)^{i}}{i !}+R_{m}(x) .
$$


Since $\psi$ has $m$ vanishing moments we have

$$
\begin{aligned}
g(x)= & W_{d} R_{m}(j, r I+x)-W_{d} R_{m}(j, s I+x) \\
= & \int_{-\infty}^{\infty} \psi\left(2^{j} t-r I-x\right) R_{m}(t) d t \\
& -\int_{-\infty}^{\infty} \psi\left(2^{j} t-s I-x\right) R_{m}(t) d t .
\end{aligned}
$$

If we let $u=2^{j} t-r I-x$ for the first integral and $u=2^{j} t-s I-x$ for the second integral we obtain

$$
\begin{aligned}
g(x)= & 2^{-j} \int_{-\infty}^{\infty} \psi(u) R_{m}\left(\frac{u+r I+x}{2^{j}}\right) d u \\
& -2^{-j} \int_{-\infty}^{\infty} \psi(u) R_{m}\left(\frac{u+s I+x}{2^{j}}\right) d u .
\end{aligned}
$$

Recall that if $f$ is expanded about $\nu$, the remainder is given by

$$
R_{m}(t)=\frac{(t-\nu)^{m}}{m !} f^{m}(\xi)
$$

for some $\xi$ between $\nu$ and $t$.

Observe that we may expand $f$ about different values of $\nu$ in separate integrals. In both cases the expansions converge to $f$. Since the wavelet transform is welldefined, changing the expansion point makes no difference in the value of $g(x)$. Letting $\nu_{r}=r I+x_{0}$ for the first integral and $\nu_{s}=s I+x_{0}$ for the second integral we obtain

$$
\begin{aligned}
g(x)= & \frac{1}{2^{j} m !} \int_{-\infty}^{\infty} \psi(u)\left(\frac{u+r I+x}{2^{j}}-r I-x_{0}\right)^{m} f^{(m)}\left(\xi_{r}(u)\right) d u \\
& -\frac{1}{2^{j} m !} \int_{-\infty}^{\infty} \psi(u)\left(\frac{u+s I+x}{2^{j}}-s I-x_{0}\right)^{m} f^{(m)}\left(\xi_{s}(u)\right) d u
\end{aligned}
$$

where $x i_{r}(u)$ is between $r I+x_{0}$ and $\frac{u+r I+x}{2^{j}}$ and $\xi_{s}(u)$ is between $s I+x_{0}$ and $\frac{u+s I+x}{2^{j}}$. If we then expand the binomials in both integrals and eliminate all terms where the exponent of $u$ is less than $m$ (by the vanishing moments of $\psi$ ) we obtain

$$
\begin{aligned}
|g(x)| & =\frac{1}{2^{j(m+1)} m !}\left|\int_{-\infty}^{\infty} \psi(u) u^{m}\left(f^{(m)}\left(\xi_{r}(u)\right)-f^{(m)}\left(\xi_{s}(u)\right)\right) d u\right| \\
& \leq \frac{\left|M_{m}\right|}{2^{j(m+1)}} 2\left\|f^{(m)}\right\|_{\infty} .
\end{aligned}
$$

The rate at which $g$ approaches a zero is bounded as follows.

Corollary 6. Let $g$ be as in Definition 2 and the wavelet $\psi$ possess $m$ vanishing moments. Then

$$
\left|g\left(t p_{k} I\right)-g_{k}\left((t+1) p_{k} I\right)\right| \leq \frac{2\left|M_{m}\right|}{2^{j(m+1)}} \sup _{\mu, \nu \in[0, P]}\left|f^{(m)}(\mu)-f^{(m)}(\nu)\right| .
$$


Proof. By the triangle inequality we have $\left|g\left(t p_{k} I\right)-g_{k}\left((t+1) p_{k} I\right)\right| \leq\left|W_{d} f\left(j, r I+t p_{k} I\right)-W_{d} f\left(j, s I+t p_{k} I\right)\right|$

$$
+\left|W_{d} f\left(j, r I+(t+1) p_{k} I\right)-W_{d}\left(j, s I+(t+1) p_{k} I\right)\right| .
$$

Each of the last two terms is bounded by

$$
\frac{\left|M_{m}\right|}{2^{j(m+1)}} \sup _{\mu, \nu \in[0, P]}\left|f^{(m)}(\mu)-f^{(m)}(\nu)\right|
$$

from the proof of Proposition 5. We restrict the supremum to the interval $[0, P]$ because $f^{m}$ has period $P$.

Corollary 7. Theorem 3 applies if we assume that only $f^{(m)}$ has period $P$ and $\psi$ is a wavelet with $m$ vanishing moments.

Proof. Since $\psi$ has $m$ vanishing moments we may write

$$
W_{d} f(j, r I+x)=\int_{-\infty}^{\infty} \psi(u) R_{m}\left(2^{-j}(u+r I+x)\right) d u .
$$

We know from Taylor's integral formula for the remainder that we may write

$$
R_{m}\left(2^{-j}(u+r I+x)\right)
$$

as

$$
\frac{1}{(m-1) !} \int_{r I+x_{0}}^{2^{-j}(u+r I+x)}\left(2^{-j}(u+r I+x)-t\right)^{m-1} f^{(m)}(t) d t,
$$

where the Taylor Polynomial is expanded about $r I+x_{0}$. Substituting this into the above expression we obtain

$$
\int_{-\infty}^{\infty} \psi(u)\left[\frac{1}{(m-1) !} \int_{r I+x_{0}}^{2^{-j}(u+r I+x)}\left(2^{-j}(u+r I+x)-t\right)^{m-1} f^{(m)}(t) d t\right] d u .
$$

Now, fix the value of $x$ at $x_{1}$ and $u$ at $u_{1}$. Then

$$
\int_{r I+x_{0}+\tau}^{2^{-j}\left(u_{1}+r I+x_{1}+2^{j} \tau\right)}\left(\frac{u_{1}+r I+x_{1}+2^{j} \tau}{2^{j}}-t\right)^{m-1} f^{(m)}(t) d t
$$

is $2^{j} P$ periodic and continuous with respect to $\tau$. This follows immediately from the periodicity and continuity of $f^{(m)}$. Also, if we subtract the above expression which corresponds to a Taylor expansion about $r I+x_{0}+\tau$ from the remainder formula at $2^{-j}\left(u+r I+x_{1}+2^{j} \tau\right)$ expanded about $r I+x_{0}$, we obtain

$$
\int_{r I+x_{0}}^{r I+x_{0}+\tau}\left(2^{-j}\left(u+r I+x_{1}+2^{j} \tau\right)-t\right)^{m-1} f^{(m)}(t) d t .
$$

Assume $r, x_{1}, x_{0}$ and $\tau$ are constants and allow $u$ to vary. Then (36) results in a polynomial in $u$ with constant coefficients of order $m-1$. By the vanishing moments of $\psi$ we may conclude that expanding $f$ about $r I+x_{0}$ and about $r I+x_{0}+\tau$ yield the same transform. Therefore, by expanding about $r I+x_{0}+\tau$ we may conclude that $W_{d} f(j, r I+x)$ is continuous and $2^{j} P$ periodic. It follows that $g(x)$ as in the definition of crossing is also $2^{j} P$ periodic and continuous. Hence the theorem holds. 
Let $g(x)$ be defined as in Definition 2. Let $n$ be the number of zeroes of $g(x)$. Pick $K$ so large that $\xi_{K}=p_{K} I-q_{K} 2^{j} P$ satisfies $\xi_{K}<\min \left\{\delta_{i}, 1 \leq i \leq n\right\}$, where $2 \delta_{i}$ are the widths of the symmetric intervals between the zeroes. Now let $f_{\tau}(x)=f(x-\tau)$. Then define

$$
\begin{aligned}
g_{\tau}(x)= & W_{d} f_{\tau}(j, r I+x)-W_{d} f_{\tau}(j, s I+x) \\
= & \int_{-\infty}^{\infty} \psi\left(2^{j} t-r I-x\right) f_{\tau}(t) d t \\
& -\int_{-\infty}^{\infty} \psi\left(2^{j} t-s I-x\right) f_{\tau}(t) d t .
\end{aligned}
$$

Let $u=t-\tau$ and obtain

$$
\begin{aligned}
g_{\tau}(x)= & \int_{-\infty}^{\infty} \psi\left(2^{j} u+2^{j} \tau-r I-x\right) f(u) d u \\
& -\int_{-\infty}^{\infty} \psi\left(2^{j} u+2^{j} \tau-s I-x\right) f(u) d u \\
= & g\left(x-2^{j} \tau\right) .
\end{aligned}
$$

So, if $g\left(x_{i}\right)=0$, then $g_{\tau}\left(x_{i}+2^{j} \tau\right)=0$. Hence the zeroes of $g$ and the symmetric intervals about each zero are translated by $2^{j} \tau$ to $\left(x_{i}+2^{j} \tau-\delta_{i}, x_{i}+2^{j} \tau+\delta_{i}\right)$. Note that the width of each interval is unchanged so that the $K$ required in the theorem is unchanged. The value of $t$ is changed for each $r$ and $s$, however. Recall that $K$ is so large that $\left|\xi_{k}\right|<\delta_{i}$ for every $1 \leq i \leq n$. Translating the function leaves the space between each pair of symmetric interval the same width, i.e., $\left[x_{i}+\delta_{i}, x_{j}+\delta_{j}\right]$ $\rightarrow\left[x_{i}+\delta_{i}+2^{j} \tau, x_{j}+\delta_{j}+2^{j} \tau\right]$.

Since the space between the intervals is unchanged we have the following propositions showing near translation invariance. Translation invariance is, of course, an important property in applications.

Proposition 8. The $t$ interval between any two crossings of $r$ and $s$ varies by at most 1 under translation.

Proof. Assume without loss of generality that $j>i$. Let $t$ be the value for which there is a crossing of the zero of $g$ at $x_{i}$. Therefore,

$$
x_{i}=t \xi_{k}+w_{i} \xi_{k}, 0 \leq w_{i}<1 .
$$

Let $t+l$ be the value for which there is a crossing at the zero of $g$ at $x_{j}$. Therefore,

$$
x_{j}=(t+l) \xi_{k}+w_{j} \xi_{k},
$$

where $0 \leq w_{j}<1$. Translating $f$ by $\tau$ translates these zeroes as indicated above. Now, $2^{j} \tau=\left\lfloor 2^{j} \tau / \xi\right\rfloor \xi_{k}+w_{\tau} \xi_{k}$, where $0 \leq w_{\tau}<1$. Call the value of $t$ after the translation $t_{\tau}$. We have $x_{i}+2^{j} \tau=t \xi_{k}+\left\lfloor 2^{j} \tau / \xi_{k}\right\rfloor \xi_{k}+\left(w_{i}+w_{\tau}\right) \xi_{k}$. Hence,

$$
t_{\tau}=t+\left\lfloor 2^{j} \tau / \xi_{k}\right\rfloor+\left\lfloor w_{i}+w_{\tau}\right\rfloor,
$$

where the last term may be 0 or 1 . The $t$ value for the crossing at $x_{j}$ is

$$
t+l+\left\lfloor 2^{j} \tau / \xi_{k}\right\rfloor+\left\lfloor w_{j}+w_{\tau}\right\rfloor,
$$

where the last term may be 0 or 1 . The difference between these values is therefore

$$
l+\left\lfloor w_{j}+w_{\tau}\right\rfloor-\left\lfloor w_{i}+w_{\tau}\right\rfloor .
$$


Note that the last difference may equal $-1,0$, or 1 . Hence the difference in $t$ value under translation is 0 or \pm 1 .

Corollary 9. Let $N_{r, s}$ be the total number of crossings between $r$ and $s$ of order $k$ in the $t$ interval given in Corollary 6 . Then $N_{r, s}$ varies by at most 1 under translation.

Proof. In Proposition 5, $i$ and $j$ were arbitrary. Hence we may take $i=1$ and $j=n$. These correspond to the first and last zeroes of $g(x)$ in the period. Let $f$ be translated by $\tau$. The $t$ interval between the crossings varies by at most 1 under translation. Since the length of the $t$ interval is greater than $2^{j} P$ and less than $2^{j} P$ plus the half the length of the smallest symmetric interval about any zero of $g$, the number of crossings per interval is $n$ or $n+1$.

Corollary 10. Let $r, s, r^{\prime}, s^{\prime}<p_{k}$. Assume without loss of generality that $r<s$ and $r^{\prime}<s^{\prime}$. If $s-r=s^{\prime}-r^{\prime}$, then $N_{r, s}=N_{r^{\prime}, s^{\prime}} \pm 1$.

Proof. The result follows upon letting $\tau=2^{-j}\left(-I\left(r^{\prime}-r\right)\right)$ and showing that $g(x)=g_{\tau}(x)$. Then apply Corollary 9 to conclude that the number of crossings under translation by $\tau$ varies by at most 1 .

Corollary 10 shows that the number of crossings depends on the difference between the translation up to 1 .

Note that if a signal $f \in L^{2}(\mathbb{R})$ is periodic on a closed interval $[a, b]$, then the above results must be adjusted to be consistent with the number of periods contained in $[a, b]$ and with nonperiodic behavior outside this interval. Here the time-frequency localization properties of wavelets are important. A continued fraction algorithm for the estimation of $M$ has been developed and will be the subject of future publication and research. Establishing the continued fraction expansion for $M$ is subject to aliasing. This problem can be addressed by an appropriate increase in the level of resolution.

\section{REFERENCES}

[1] C. Burrus, R. Gopinah, and H. Guo, Introduction to Wavelets and Wavelet Transforms: A Primer, Prentice-Hall, Inc., Upper Saddle River, New Jersey, 1998, pp. 190-195.

[2] W. LeVeque, Fundamentals of Number Theory, Addison-Wesley Publishing Company, Inc., 1977, pp. 232-237. MR 58:465

[3] S. Mallat, A Wavelet Tour of Signal Processing, Academic Press, New York, 1998, pp. 169171. MR 99m:94012

[4] Ya. Khintchine, Continued Fractions, P. Noordhoff Ltd., Groningen, 1963, pp. 11-12. MR 28:5038

Department of Mathematics and Computer Science, Colorado College, Colorado Springs, Colorado 80903

E-mail address: jglenn@coloradocollege.edu 